\title{
Determinação do comportamento de codificadores em diferentes condições de rede em um cenário experimental
}

\author{
Demóstenes Zegarra Rodriguez, Miguel Arjona Ramírez
}

\begin{abstract}
Resumo - Perda de pacotes e atrasos influenciam na qualidade de uma conversa interativa em uma rede IP. Este artigo mostra uma metodologia para predizer o comportamento de diferentes codificadores, utilizando modelos objetivos como o PESQ e a Recomendação E-Model do ITU-T. A metodologia apresentada é útil para medir a performance de novos codificadores, ou de codificadores que ainda não possuam parâmetros definidos em diferentes cenários de perda de pacotes e atrasos, em uma comunicação bidirecional utilizando uma rede emulada com open source software, sendo o cenário facilmente reproduzido.
\end{abstract}

Palavras-Chave - Voz sobre IP, MOS, codificador, EModel, PESQ.

Abstract - Packet loses and delays have influence in the quality of an interactive voice conversation in an IP network. This article exhibits a methodology to predict the behavior of different codecs using objectives models as PESQ and E-Model Recommendation from ITU-T. This methodology is useful to measure the performance of new codecs, or that do not have defined parameters in different scenarios of packet loses and delays in a bidirectional communication using a network emulated with software open source, where the scenario can be easily reproduced.

Keywords - VoIP, MOS, codec, E-Model, PESQ.

\section{INTRODUÇÃO}

$\mathbf{N}$ projeto de redes de pacotes devem-se considerar diversos parâmetros como: perda de pacotes, atrasos fixos e variáveis, taxa de codificação, taxa total de transmissão e o eco [1], [2], [3] e [4] que afetam diretamente a qualidade da transmissão e, conseqüentemente, a qualidade do sinal no receptor.

É necessário que o projetista de rede conheça em que medida cada parâmetro afeta a qualidade em uma comunicação. Este trabalho está orientado ao estudo do fator de degradação do codificador definido na recomendação ITU-T G.107 [5], mais conhecida como: E-model. A recomendação ITU P.862

Laboratório de Processamento de Sinais da Universidade de São Paulo, Brasil, E-mails: demostenes@lps.usp.br, miguel@lps.usp.br

Este trabalho foi financiado parcialmente pela Fundação de Amparo à Pesquisa do Estado de São Paulo (FAPESP) através dos processos 2007/08288-2 e 2004/13206-7 e pela Coordenação de Aperfeiçoamento de Pessoal de Nível Superior (CAPES) através de uma Bolsa de Mestrado.
[6] geralmente conhecida como PESQ (perceptual evaluation of speech quality), em termos gerais, descreve um método objetivo para avaliar a qualidade subjetiva de codificadores. É importante considerar que este algoritmo estabelece uma avaliação precisa em comunicações unidirecionais, mas não permite medir a qualidade da transmissão em uma comunicação bidirecional, sendo possível obter resultados altos de qualidade mesmo com uma conexão global ruim, assim, por exemplo, o atraso ponto a ponto em uma rede de transmissão de pacotes afeta a qualidade de voz somente nas comunicações bidirecionais.

No caso de uma transmissão em uma rede ideal (sem perdas e nem atrasos) ou que apresente somente perda de pacotes, os resultados do PESQ são confiáveis e estes podem ser utilizados junto com a Recomendação ITU-T G.107 para calcular o parâmetro que corresponde às características próprias de cada codificador, e que é denominado nesta recomendação como Ie, fator de degradação do codificador. Conhecendo este parâmetro, pode-se estimar o comportamento do codificador em estudo em diferentes cenários de atraso e perdas de pacotes.

Na Recomendação ITU G.108 [7] é apresentada uma explicação de como se devem interpretar as equações da Recomendação ITU-T G.107 apresentando-se alguns casos práticos que mostram como este método é corretamente utilizado. Na recomendação ITU- T G.113 Anexo I [8] são apresentados os valores de Ie para diferentes codificadores que já foram estudados pela ITU-T, estes valores são obtidos de maneira experimental e são atualizados periodicamente.

Portanto, neste artigo se propõe fazer medidas experimentais com os codificadores de voz Rec. ITU-T G.723.1 [9] e iLBC [10] em um cenário de rede emulada e utilizando a ferramenta PESQ. para estimar o índice MOS (mean opinion score) descrito na Recomendação ITU-T P.800 [11] para diferentes cenários de probabilidade de perda de pacotes (incluindo perda de pacotes igual a zero). Com estes valores obtidos e empregando as equações do E-Model que relacionam o valor do índice MOS com o valor de determinação do índice de transmissão R, pode-se calcular o parâmetro Ie do codificador em estudo.

A metodologia seguida para estes codificadores é aplicável para os codificadores novos que ainda não tenham parâmetros definidos e a partir destes, fazer uma estimativa de sua qualidade em diferentes cenários de probabilidade de perda de pacotes e em diferentes tempos de atraso em comunicações bidirecionais.

Este artigo é inovador pelo fato da utilização de ferramentas 
open source que podem ser facilmente reproduzidas e assim possibilitar a comprovação de resultados e permitir que se obtenha o parâmetros Ie de novos codificadores que estão sendo implementados, estimulando o surgimento destes.

$\mathrm{O}$ artigo está dividido respectivamente em: Seção 2, onde é feito um resumo teórico das ferramentas utilizadas. Seção 3 que apresenta o cenário onde são realizados os testes indicando a metodologia seguida e os parâmetros avaliados. Seção 4, onde são apresentados e discutidos os resultados obtidos, e por fim, a Seção 5 que expõe a importância da determinação do fator de degradação do codificador descrito no E-Model em um cenário de rede experimental.

\section{REVISÃO TEÓRICA}

Os métodos utilizados neste trabalho foram o método intrusivo, ou seja, que requer dois sinais de referência, PESQ e o método não intrusivo, que só requer de um sinal, E-Model.

O PESQ é uma ferramenta objetiva na avaliação da qualidade de voz, utilizando como entradas no seu algoritmo, dois sinais: o sinal original e o sinal degradado.

O E-model é um modelo computacional que mede os efeitos da variação dos parâmetros de uma rede de transporte e do sinal na qualidade de voz transmitida.

O E-Model é definido pela seguinte equação:

$$
\mathrm{R}=\mathrm{Ro}-\mathrm{Is}-\mathrm{Id}-\mathrm{Ie}+\mathrm{A}
$$

Onde:

- R: fator de determinação do índice de transmissão, representa o parâmetro que está relacionado com o valor de qualidade e tem uma correspondência com a pontuação MOS UIT-T P.800.

- Ro: relação sinal-ruído.

- Is: fator de degradações simultâneas, representa todas as degradações que se produzem simultaneamente com o sinal de fala, como são os níveis dos sinais de fala de maior intensidade (OLR não ótima), o efeito local não ótimo (STMR) e o ruído de quantificação.

- Id: degradação da qualidade causada devido ao atraso existente na rede.

- Ie: degradação da qualidade por efeito do equipamento (codificador).

- A: fator de melhora, não tem relação com os demais parâmetros da transmissão.

O valor padrão de Ro é 93.2 [5], que é obtido ao se colocar todas as entradas do modelo com seus valores padrões. $\mathrm{O}$ parâmetro Is não será considerado nos cálculos, já que este descreve as condições que são relacionados com o sinal, não dependendo da rede de transporte. O fator A, segundo [5] tem o valor 0 (zero) para redes com fio e é o que corresponde ao cenário de emulação deste trabalho.

O fator Id é definido como:

$$
\mathrm{Id}=\text { Idle }+ \text { Idd }
$$

Os fatores Idte e Idle correspondem ao atraso causado pelo eco no emissor e no receptor, respectivamente. Estes fatores não serão considerados neste trabalho já que para o cenário de testes se assumirá uma supressão perfeita do eco.

O Idd representa os atrasos produzidos na rede e no codificador. $\mathrm{O}$ atraso ocasionado pelo codificador se define em [3] como:

$$
\text { Idd }=2 \mathrm{~N}+\text { Pré-análise }
$$

Onde $\mathrm{N}$ é o tamanho da amostra de cada codificador. Valores de $\mathrm{N}$ e dos tempos de pré-análise para diferentes codificadores podem ser encontrados em [12]. Com estas considerações, pode-se calcular o parâmetro $\mathrm{R}$ em função dos parâmetros: atraso (Id) e o fator correspondente ao codificador (Ie):

$$
\mathrm{R}=\mathrm{Ro}-\mathrm{Id}-\mathrm{Ie}
$$

\section{CENÁRIO EXPERIMENTAL}

O seguinte diagrama de blocos, Figura 1, ilustra o cenário experimental utilizado para encontrar o parâmetro de degradação do codificador Ie, empregando as recomendações descritas anteriormente.

Os codificadores de voz que foram avaliados em um cenário sem perdas e sem atrasos foram: G723.1 e iLBC.

Para o codificador G.723.1 foram feitos testes com probabilidades de perdas de pacotes de $0 \%, 5 \%, 10 \%$ e $20 \%$ com atraso ponto a ponto igual a zero.

Este cenário é composto por uma sub-rede (PC1) conectado a uma outra sub-rede (PC3), e estes são interconectados por um roteador GNU/Linux (PC2). Sendo utilizada uma rede com fio para este cenário.

Os softwares aplicativos utilizados nos testes foram:

- PC1, PC3: clientes com softphone MyPhone 0.2b10 [13], analisador de pacotes Wireshark [14] e software para gravação de áudio .wav VRS Recording System [15].

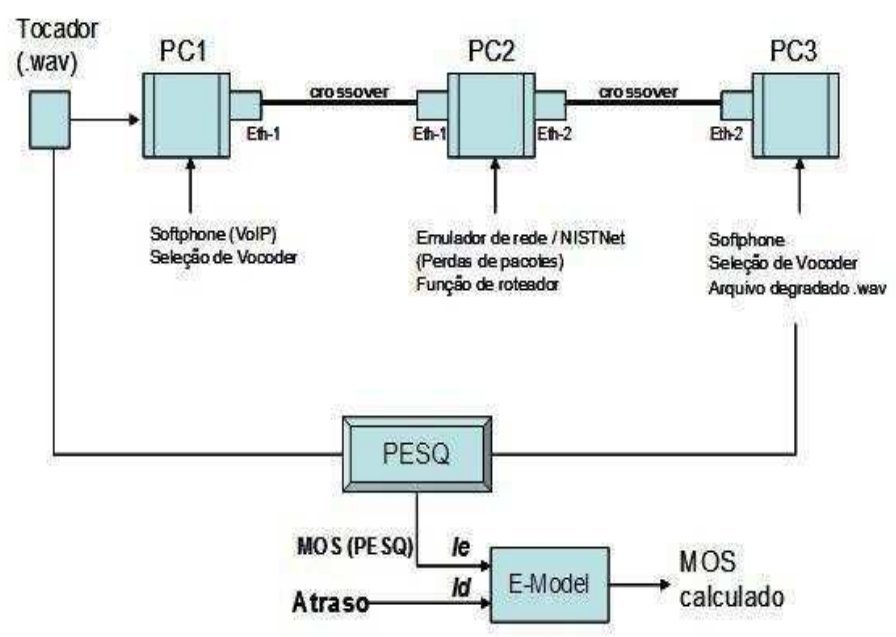

Fig. 1. Diagrama de blocos do cenário de testes utilixando ferramentas open source 
TABELA I

- PC2: roteador com o emulador de redes Nistnet [16] utilizado para simular perda de pacotes, atrasos, variações de atrasos e largura de banda e o software ITU-T P.862 utilizado para encontrar o índice MOS da qualidade de voz.

O som transmitido, foi gerado por um reprodutor de arquivo .wav que é conectado a entrada do microfone do PC1 mediante um cabo de áudio. Este arquivo possui uma duração de 8 segundos e foi amostrado a $8 \mathrm{kHz}$ e 16 bits.

A Metodologia seguida nos testes para se obter o valor MOS utilizando a ferramenta PESQ é a seguinte:

- Inicialmente, se inicia uma comunicação entre o PC1 e o PC3 mediante os softphones que cada PC possui instalado e que permite escolher o codificador de voz utilizado para cada chamada VoIP, onde esta é feita de computador a computador, e a sinalização utilizada foi a recomendação H.323.

- Para cada cenário o emulador de rede é configurado com os parâmetros requeridos do teste a executar.

- O reprodutor transmite o áudio (arq-orig.wav) ao PC1 onde este som é gravado (arq-orig2.wav), como a chamada já está ativa, a voz é codificada e transmitida para o PC3 através do PC2.

- Enquanto os dados são transmitidos, o programa Wireshark que está sendo executado no PC1 e no PC3, salva a informação da rede como: as mensagens de sinalização para estabelecimento, manutenção e finalização das chamadas, as mensagens do protocolo RTP, o tamanho médio do pacote (Bytes), numero médio de pacotes transferidos por segundo e largura de banda média.

- No PC3, o áudio recebido é gravado em um arquivo (arq-deg.wav). Este arquivo e o arquivo 'arq-orig2' são comparados mediante o programa PESQ, que é executado no PC2, tendo como resultado uma pontuação MOS. Foram feitos dez testes para cada cenário, tomando-se o valor médio como resultado válido de cada cenário. $\mathrm{O}$ desvio padrão obtido pelo programa PESQ foi de 0,05 para cada codificador.

É importante mencionar que antes de realizar cada teste, o programa PESQ foi configurado adequadamente. Neste sentido, verificaram-se todos os arquivos de teste que existentes no software PESQ, obtendo-se resultados satisfatórios.

Da mesma forma para os cenários de perdas de pacotes e atrasos, configurados no Nistnet, foram feitos testes para avaliar se estes atrasos e perdas eram reflexos de dados anteriores da rede a fim de se obter um resultado confiável.

\section{RESULTADOS}

\section{A. Cenário sem probabilidade de perda de pacotes}

Para um cenário ideal: sem perdas, sem atrasos e com taxa de transmissão suficiente, conseguiram-se resultados semelhantes com a maioria da literatura como em [17], [18] e [19].

A Tabela I apresenta as médias dos valores obtidos nos testes.

Segundo [20] estes valores MOS podem ser convertidos em valores MOS-LQO (Mean Opinion Score - Listening Quality
ÍNDICE MOS PARA OS CODIFICADORES G.711, G.723.1 E ILBC EM UM

CENÁRIO SEM PROBABILIDADE DE PERDAS DE PACOTES E SEM ATRASOS.

\begin{tabular}{cc}
\hline Codificador & Pontuação PESQ \\
\hline G.711 & 4.09 \\
G.723.1 & 3.52 \\
iLBC & 3.79 \\
\hline
\end{tabular}

Objective), que é a pontuação do PESQ mais comparável com o índice MOS pela seguinte equação:

Sendo MOS $=\mathrm{x}$ e para $\mathrm{x}>1.7$ :

$$
\begin{aligned}
& \text { MOS-LQ }=-0.156278 \mathrm{x}^{3}+1.386609 \mathrm{x}^{2}-2.504699 \mathrm{x} \\
& +2.023345
\end{aligned}
$$

Para MOS $\leq 1.7:$

$$
\text { MOS-LQ }=1
$$

Com estes valores e utilizando a equação 6 definida em [9], pode determinar-se o valor de $\mathrm{R}$ a partir do índice MOS.

Para $\mathrm{MOS}=1.0$ :

$$
\mathrm{R}=0
$$

Para $1.0<\operatorname{MOS}<4.5$ :

$$
\begin{aligned}
& \text { MOS-LQ }=1+0.035 \times \mathrm{R}+\mathrm{R} \times(\mathrm{R}-60) \times 7 \times 10-^{6}(8) \\
& \text { Para MOS } \geq 4.5:
\end{aligned}
$$

$$
\mathrm{R}=100
$$

E para se obter o parâmetro Ie utilizou-se a equação 10 , considerando Id=0 e Ro igual ao valor obtido para o codificador da recomendação ITU-T G.711 [21] no cenário de rede de testes apresentado.

$$
\mathrm{Ie}=86.5-\mathrm{R}
$$

Com estas equações obtiveram-se os valores apresentados na Tabela II:

TABELA II

VALORES DE IE, PESQ E PESQ-LQO PARA OS CODIFICADORES G.723 E ILBC.

\begin{tabular}{cccc}
\hline Codificador & PESQ & PESQ-LQO & Ie \\
\hline G.723.1 & 3.52 & 3.53 & 18 \\
iLBC & 3.79 & 3.88 & 13 \\
\hline
\end{tabular}

Da Tabela II, pode-se observar que o valor do Ie obtido para o codificador iLBC apresenta um melhor resultado segundo a equação 2 , onde um menor valor de degradação (Ie) reflete uma maior qualidade do sinal. 
É importante ressaltar que o valor obtido para o $\mathrm{Ie}=18$ do codificador G.723.1 é muito próximo ao valor apresentado pela ITU-T no anexo I da Rec. G.113 que é igual a 19.

Com os valores da Tabela II para o codificador G723.1 obteve-se o gráfico da Figura 2, que apresenta a relação entre o MOSc (MOS conversacional) obtido do valor R do E-Model e os diferentes valores de atraso $(0,50,100,150,200,250$, $300,350,400,450$ e $500 \mathrm{~ms}$ ) para um cenário de perdas de pacotes igual a zero.

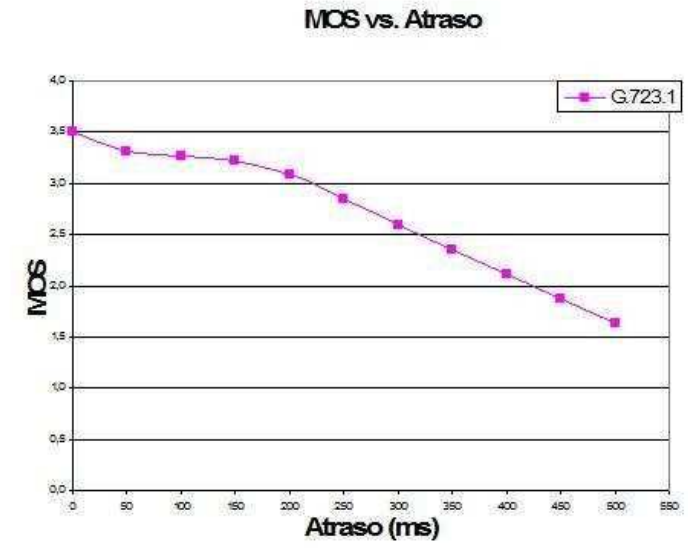

Fig. 2. Cálculo de MOS vs. Atraso obtido utilizando o E-model

Desta última figura, pode-se constatar que a qualidade de voz se conserva aceitável até $150 \mathrm{~ms}$ que é o atraso especificado na Recomendação ITU-T G.114 [22].

\section{B. Cenário com probabilidade de perdas de pacotes}

Para um cenário de probabilidade de perdas de pacotes e atrasos iguais a zero, e seguindo a metodologia explicada, mediu-se a qualidade de voz para os codificadores G.723.1 e iLBC.

As probabilidades de perdas consideradas nos testes realizados foram de $5 \%, 10 \%$ e $20 \%$.

Os resultados obtidos são apresentados na Tabela III.

TABELA III

VALORES MOS E IE PARA OS CODIFICADORES G.723.1 E ILBC EM UM

CENÁRIO COM PERDAS DE PACOTES.

\begin{tabular}{ccc}
\hline & \multicolumn{1}{c}{$\begin{array}{c}\text { Codificador } \\
\text { G.723.1 }\end{array}$} & \multicolumn{1}{c}{$\begin{array}{c}\text { Codificador } \\
\text { iLBC }\end{array}$} \\
\hline & $\begin{array}{l}\text { Probabilidade de } \\
\text { perda de pacotes }\end{array}$ & $\begin{array}{l}\text { Probabilidade de } \\
\text { perda de pacotes }\end{array}$ \\
\hline MOS & $5 \%-10 \%-20 \%$ & $5 \%-10 \%-20 \%$ \\
Ie & $2.85-2.60-2.14$ & $3.26-3.02-2.61$ \\
\hline
\end{tabular}

O codificador iLBC em relação com o codificador G.723.1 teve uma melhor resposta ante perdas de pacotes, o que pode comprovar-se com os novos valores do fator Ie, obtidos para cada um dos codificadores nos diferentes cenários apresentados na Tabela III (Quanto menor Ie melhor resposta à perda de pacotes)
A resposta de um codificador ante diferentes cenários de perda de pacotes se denomina grado de Robustez do codificador e esta definida pela seguinte equação:

$$
\text { Ie-eff }=\text { Ie }+(95-\mathrm{Ie}) \times(\mathrm{Ppl} /(\mathrm{Ppl} / \mathrm{BurstR}+\mathrm{Bpl})
$$

Onde:

- Ie-eff: Valor de Ie para um determinado cenario de perda de pacotes.

- Ie: Valor de Ie num cenário ideal.

- Ppl: Probabilidade de perda de pacotes.

- BurstR: Relação de rajada.

- Bpl: Fator de Robustez contra perda de pacotes.

E considerando que:

- Lmc = Longitude média das rajadas observadas em uma seqüência de chegada;

- Lma = Longitude média das rajadas previstas na rede em condições de perdas arbitrárias.

Por sua vez o parâmetro BurstR é definido como:

$$
\text { BurstR }=\text { Lmc/Lma }
$$

Com perda de pacotes aleatória o valor de Burst será 1 e quando as perdas de pacotes obedeçam às rajadas, ou seja, que são dependentes, o valor de Burst será maior que 1. Para perda de pacotes que sigam o modelo de Markov com dos estados, se tem:

$$
\text { BurstR }=1 /(\mathrm{p}+\mathrm{q})=(\mathrm{Ppl} / 100) / \mathrm{p}
$$

Sendo p e q as probabilidades de transição entre pacotes localizados e pacotes de perda.

TABELA IV

VALORES DE ROBUSTEZ CONTRA PERDA DE PACOTES PARA OS CODIFICADORES G.723.1 E ILBC

\begin{tabular}{ccc}
\hline G.723.1 & 1LBC & \\
\hline Ie & 18 & 13 \\
Ppl & 5 & 5 \\
BurstR & 1 & 1 \\
Ie-eff & 37 & 26 \\
Bpl & 15.26 & 26.54 \\
\hline
\end{tabular}

A Tabela IV apresenta os valores de Robustez contra perda de pacotes para os codificadores G.723.1 e iLBC.

Observando-se um maior valor de Bpl para o codificador iLBC.

\section{CONCLUSÕES}

Este trabalho pretende ser útil para projetistas de rede na avaliação do valor de Ie de codificadores novos ou que não tenham parâmetros definidos pela ITU-T, e a partir deste predizer o índice de qualidade MOS do codificador, em estudo para diferentes cenários de rede, ou seja, com diferentes probabilidades de perda de pacotes e atrasos tanto em conversações unidirecionais quanto em conversações bidirecionais. 
Os resultados obtidos foram bastante aceitáveis considerando que são valores muito próximos aos descritos na documentação da ITU-T, dando um grau de confiabilidade ao método utilizado em estes testes e que podem ser facilmente reproduzíveis já que o cenário de teste foi baseado em software open source.

\section{TRABALHOS FUTUROS}

Neste trabalho foi descrito uma metodologia para predizer o comportamento de um codificador em diferentes cenários de redes; estes resultados podem ajudar na análise da rede de um modo estático e em momentos determinados com as modificações nos diversos elementos da rede, especialmente na eleição da taxa de codificação que só poderá ser feita manualmente. Como o comportamento das redes de dados é variável, a melhor solução é projetar um sistema que controle a qualidade da comunicação de uma forma dinâmica e automática. Considerando isto em um trabalho futuro pretende-se criar um algoritmo para a determinação da taxa de codificação (RDA) que ajude a controlar automaticamente a qualidade do sistema, ou seja, para condições de rede com sobrecarga de tráfego, a taxa a ser escolhida será sempre a menor e para condições de baixa carga da rede a taxa escolhida será a maior.

\section{REFERENCES}

[1] M. Bathia, S. Kalindrini, Voice over IP Fundamentals, Second Edition, CiscoSystems, Aug. 2007.

[2] J. Han, J. Chung, Study of delay patterns of weighted voice traffic of end to-end users on the VoIP Networks, International Journal of Network Management, 2002.

[3] A. P. Markopoulou, F. A. Tobagi, Assessment of VoIP Quality over Internet, in Proc. of IEEE Infocom, vol. 1, (New York, USA), pp. 150159 , June 2002.

[4] D. Minoli and E. Minoli, Delivering Voice over IP Networks, 2nd Edition. John Wiley and Sons, 2002.

[5] ITU-T Rec. G.107, The E-model, a computational model for use in transmission planning, Mar. 2005. http://www.itu.int/rec/T-REC-G.107/en.

[6] ITU-T Rec. P.862, Perceptual evaluation of speech quality (PESQ): An objective method for end-to-end speech quality assessment of narrowband telephone networks and speech codecs, http://www.itu.int/rec/TREC-P.862/en.

[7] ITU-T Rec. P.G.108, Aplicación del modelo E: Directrices para la planificación, Set. 1999. http://www.itu.int/rec/T-REC-G.108/en.

[8] ITU-T Rec. G.113, Degradaciones de la transmisión debido al tratamiento de los señales vocales, May. 2002. http://www.itu.int/rec/T-RECG.113/en.

[9] ITU-T Rec. G.723.1, General Aspects of Digital Transmission Systems Dual Rate Speech Coder for Multimedia Communications Transmitting at 5.3 and $6.3 \mathrm{kbit} / \mathrm{s}$, May. 2006. http://www.itu.int/rec/T-REC-G.723.1/en.

[10] IETF RFC 3951, Internet Low Bit Codec Rate, Dec. 2004. http://www.ietf.org/rfc/rfc3951.txt.

[11] ITU-T Rec. P.800, Methods for subjective determination of transmission quality, Aug. 1996. http://www.itu.int/rec/T-REC-P.800/en.

[12] M. Arjona, M. Minami, Technology and Standards for Low-Bit-Rate Voding Methods, The Handbook of Computer Networks. 1 ed. Hoboken, N.J.: John Wiley Sons, 2008, v. 2, p. 447-467.

[13] Softphone Myphone, http://myphone.sourceforge.net/.

[14] Wireshark, http://www.wireshark.org/download.html.

[15] VRS - Recording System, http://www.nch.com.au/vrs/index.html.

[16] NISTNET, http://www-x.antd.nist.gov/nistnet/.

[17] M. Jiang, M. Schulzrinne, Comparisons of fec and codec robustness on voip quality and brandwidht efficiency, Columbia University, Department of computer Science, USA.

[18] Voice over packet, An assessment of voice performance on packet networks, Nortel Networks. 2002.

[19] M. Grant, S. Tonissen, $\quad$ Voice Quality Monitoring for VoIP Networks, Calyptech. 2003.
[20] A. W. Rix, Comparison between Subjective Listening Quality and P.862 PESQ Score, in Proceedings of Online Workshop Measurement of Speech and Audio Quality in Networks, (Czech Republic), May 2003.

[21] ITU-T Rec. G.711, General Aspects of Digital Transmission Systems Terminal Equipments - Pulse Code Modulation (PCM) of Voice Frequencies, 1972. http://www.itu.int/rec/T-REC-G.711/en.

[22] ITU-T Rec. G.114, One way transmission time, May. 2003. http://www.itu.int/rec/T-REC-G.114/en. 